\title{
A Hilbert Space Interpretation of the Power Theory
}

\author{
Roberto Garcia-Rochin
}

\begin{abstract}
The definition of the reactive power in nonsinusoidal conditions has been a controversial topic since the experiment published by C. P. Steinmetz in 1892. Where among Electrical Engineers there is no consensus on how to compute the reactive power in non-sinusoidal conditions. Therefore, in this paper it is used the mathematical concept of a Hilbert space, namely the $L_{2}$ and $\ell_{2}$ spaces, and the Least effort problem to obtain the definition of the generalized reactive power. Then, the obtained definition of the generalized reactive power is compared against some of the main power theories. Finally, it is used that definition to study the relation between the Tellegen's theorem with the Least effort problem and a novel application is presented.
\end{abstract}

Index Terms-Hilbert spaces, Power measurement, Reactive power, Vectors, Functional analysis, Optimization.

\section{INTRODUCTION}

$\mathbf{I}$ N 1892 C.P. Steinmetz published an experiment of a singlephase Alternating Current (AC) electric arc, that showed a non-sinusoidal behaviour of the voltages and currents. In that experiment, he computed the apparent and active power of the non-sinusoidal load, and even though the current and voltage were in phase, the apparent power was bigger than the active power. That result had as a consequence the question, Does a phase shift occur in an AC arc? [1]. This pitfall of the Classical power theory lead to the development of several theories attempting to fully understand that phenomena. In 1927, C. Budeanu attempted to describe this phenomena by using the complex Lagrange's identity to compute the reactive power generated in non-sinusoidal conditions for a singlephase circuit [2]. Budeanu's power theory was developed in the so called frequency domain, because it is using the harmonic components of the voltages and currents to compute the powers of the signals. In 1931, S. Fryze obtained the current that generates no reactive power for a single-phase circuit, by using the Cauchy-Schwarz inequality [3]. Because Fryze used the voltages and currents as a function of time, this theory is called to be in the time domain. The similarity between these two theories is that they are computed over the whole period of the signals, thus they do not give an instantaneous result. To cope with that, H. Akagi et. al. developed the instantaneous power theory [4], that is used to compensate for the reactive power in three phase systems under balanced sinusoidal conditions [5]. These three theories attempted to compute the reactive power. However, they did not give a meaning to that quantity. But in 1985, the Institute of Electrical Circuit Theory and Engineering defined the reactive power problem as an optimization problem with constrains [6]. Where the objective function is the Root Mean Squared (RMS)

R. Garcia-Rochin is with the Electrical Energy Systems Group, Department of Electrical Engineering, Eindhoven University of Technology, $5600 \mathrm{MB}$ Eindhoven, The Netherlands (e-mail: r.garcia.rochin@tue.nl). value of the current and the constrain is to keep the active power as a constant. By solving this optimization problem it is obtained the current that generates no reactive power described by Fryze [6].

These four theories described above are the basis for most of the developed power theories, since they rely on computations in the frequency domain [7]-[10], time domain [11], [12] or as an instantaneous computation [13], [14].

The lack of consensus among Electrical Engineers on the meaning and how to compute the reactive power lead to the IEEE Std. 1459-2000 [15]. That standard attempted to unify the definition of the reactive power among the Electrical Engineers [15]. But due to more debates, the standard was changed in 2010 [16].

The debate on the definition of the reactive power continues up to these days. Where none of the theories given above fully describe the puzzle of the power theory, due to the lack of a proper mathematical framework.

In this work, it is used the concept of a real Hilbert space as the mathematical framework to give a generalized definition of the reactive power. A similar framework has been used before to describe the reactive power problem [17], [18]. In [17], the authors used the geometrical properties of a finite dimensional vectors space to derive a definition of the reactive power, here it is used an infinite dimensional vector space. In [18], they used the notion of projections in a Hilbert space to derive the reactive power, but they did not made a difference between real and complex Hilbert spaces. The difference between this work and [18] is that here we begin solely with the definition of a real Hilbert space and its geometrical properties, and from that it is constructed the definition of the generalized reactive power. With this new definition, the reactive power is interpreted as a geometrical property of the real Hilbert space, and the computations done in AC circuits are extended to switched Direct Current (DC) circuits.

This paper is organized as follows. The mathematical preliminaries are given in Section II. In Section III, it is derived the definition of the generalized reactive power, and with that definition it is obtained the reactive power for a multiphase system. Section IV gives a comparison of some power theories with the derived definition of the reactive power. A criticism of the application of the Tellegen's theorem in AC systems using complex Hilbert spaces is presented in Section V. Finally, an example is given in Section VI, and the conclusions are drawn in Section VII.

\section{HILBERT SPACES}

The Hilbert space $\mathcal{K}$ can be seen as the generalization of the Euclidean space, but the number of vectors that are needed to span the space can be infinite [19]-[21]. The linear space 
$\mathcal{K}$ is equipped with an operation called inner product [19][21]. That operation is defined between two vectors $\hat{x}, \hat{y} \in \mathcal{K}$ as the ordered pair $\langle\hat{x}, \hat{y}\rangle$, where the result is a scalar [19][21]. The result of that inner product will define if $\mathcal{K}$ is a real or a complex Hilbert space. With the definition of the inner product, it is induced the norm of the vector $\hat{x}$ by (1) [21],

$$
\|\hat{x}\|=\sqrt{\langle\hat{x}, \hat{x}\rangle} .
$$

This operation defines the notion of the length of the vector $\hat{x}$. Another quantity that is defined when $\mathcal{K}$ is a real Hilbert space, is the angle $\phi$ between the two vectors $\hat{x}, \hat{y} \in \mathcal{K}[20]$, [21],

$$
\cos \phi=\frac{\langle\hat{x}, \hat{y}\rangle}{\|\hat{x}\|\|\hat{y}\|} .
$$

From (2) it is said that the vectors $\hat{x}$ and $\hat{y}$ are orthogonal if $\langle\hat{x}, \hat{y}\rangle=0$ [20], [21]. Also, the Cauchy-Schwarz inequality (3) is a property of this space,

$$
\langle\hat{x}, \hat{y}\rangle \leq\|\hat{x}\|\|\hat{y}\| .
$$

Where this inequality becomes an equality if $x=\alpha y$ or if one of the vectors is the zero vector [20], [21]. Another important property is that it is defined a notion of a projection of one vector onto another [20], [21].

In this work, it will be defined the Least-effort problem for a real Hilbert space. Then, the quantities defined above (1)-(3), along with the term needed to convert the CauchySchwarz inequality into an equality and a basis to span the linear space are given for the real $L_{2 \mathbb{R}}^{n}[a, b]$ space. After that, it is used the notion of isomorphism and complexification of Hilbert spaces to generate the real $\ell_{2 \mathbb{R}}^{1}$, complex $L_{2 \mathbb{C}}^{1}[a, b]$ and complex $\ell_{2 \mathbb{C}}^{1}$ spaces from $L_{2 \mathbb{R}}^{n}[a, b]$. These Hilbert spaces were chosen because they can describe periodic functions.

\section{A. Least Effort Problem}

The Least effort problem, is an optimization problem that looks for the minimum norm of the vector $\hat{x} \in \mathcal{H}$, subject to constrains given by inner products with the vectors $\hat{y}_{1}, \hat{y}_{2}, \ldots, \hat{y}_{k} \in \mathcal{H}[20]$. In this work the Least effort problem is defined as an abstract variational problem in a real Hilbert space $\mathcal{H}$. This was done because we want to use the notion of an angle defined in (2). Then, this optimization problem is given as,

$$
\begin{array}{ll}
\underset{\hat{x} \in \mathcal{H}}{\operatorname{minimize}} & \|\hat{x}\|^{2}, \\
\text { subject to } & \left\langle\hat{x}, \hat{y}_{p}\right\rangle=c_{p} \in \mathbb{R} \quad p=1, \ldots, k .
\end{array}
$$

The solution of this norm minimization problem is achieved by following Theorem 1 [21, p. 65].

Theorem 1: Let the set of linearly independent vectors $\hat{y}_{1}, \hat{y}_{2}, \ldots, \hat{y}_{k} \in \mathcal{H}$ span a subspace $\mathcal{M} \subset \mathcal{H}$. Therefore, among all vectors $\hat{x} \in \mathcal{H}$ that satisfy,

$$
\left\langle\hat{x}, \hat{y}_{p}\right\rangle=c_{p} \quad p=1, \ldots, k .
$$

And letting $\hat{x}_{0}$ be the minimum norm vector of $\hat{x}$. Then,

$$
\hat{x}_{0}=\sum_{p=1}^{k} \beta_{p} \hat{y}_{p} .
$$

Where the constants $\beta_{1}, \beta_{2}, \ldots, \beta_{k}$ are obtained by solving the system of equations (7), known as the Gram equations [20],

$$
\left(\begin{array}{cccc}
\left\langle\hat{y}_{1}, \hat{y}_{1}\right\rangle & \left\langle\hat{y}_{2}, \hat{y}_{1}\right\rangle & \ldots & \left\langle\hat{y}_{k}, \hat{y}_{1}\right\rangle \\
\left\langle\hat{y}_{1}, \hat{y}_{2}\right\rangle & \left\langle\hat{y}_{2}, \hat{y}_{2}\right\rangle & \ldots & \left\langle\hat{y}_{k}, \hat{y}_{2}\right\rangle \\
\vdots & \vdots & \ddots & \vdots \\
\left\langle\hat{y}_{1}, \hat{y}_{k}\right\rangle & \left\langle\hat{y}_{2}, \hat{y}_{k}\right\rangle & \ldots & \left\langle\hat{y}_{k}, \hat{y}_{k}\right\rangle
\end{array}\right)\left(\begin{array}{c}
\beta_{1} \\
\beta_{2} \\
\vdots \\
\beta_{k}
\end{array}\right)=\left(\begin{array}{c}
c_{1} \\
c_{2} \\
\vdots \\
c_{k}
\end{array}\right)
$$

The proof of Theorem 1 is given in [21, p. 66]. Because that proof is important to understand the reactive power problem, it is described here. Looking at (5), it can be seen that the constrains define hyperplanes in $\mathcal{H}$. Thus, their intersection result in the hyperplane $\mathcal{V}$. In order to find the minimizing vector $\hat{x}_{0}$ we must obtain an orthogonal vector to $\mathcal{V}$. Since the subspace $\mathcal{M}$ is orthogonal to the hyperplane $\mathcal{V}$. The minimizing vector $\hat{x}_{0}$ is a linear combination of the vectors describing $\mathcal{M}$, as we can see in (6). Finally, it is obtained the intersection between $\mathcal{M}$ and $\mathcal{V}$ with the substitution of (6) into (5), leading to the Gram equations (7). A geometrical interpretation of the proof of Theorem 1 in the $\mathbb{R}^{3}$ space with two hyperplanes as constrains is depicted in Fig. 1.

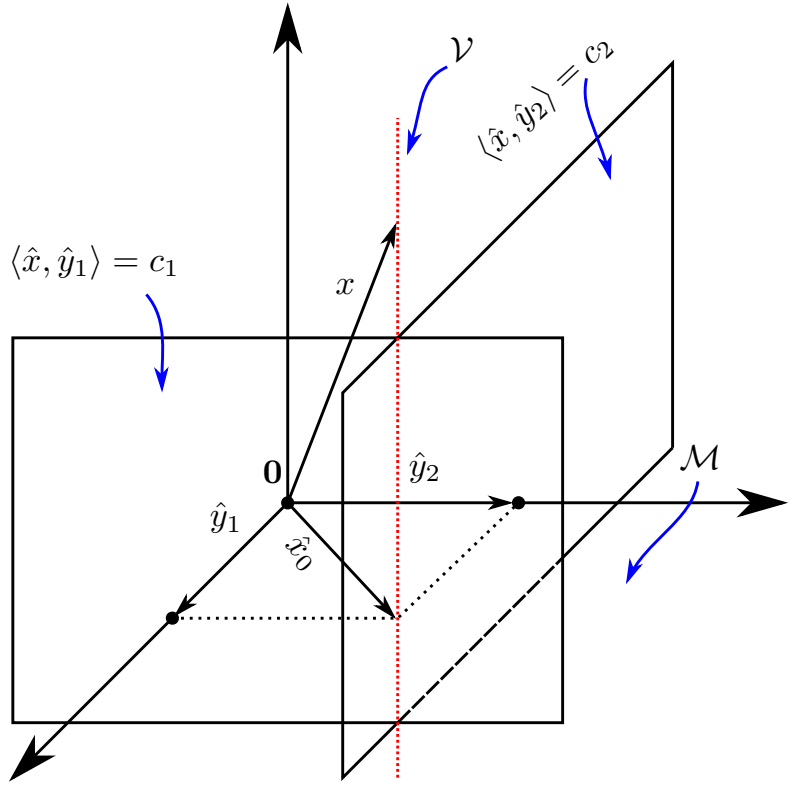

Fig. 1. Geometrical interpretation of the proof of Theorem 1 [20].

The Least-effort problem (4) was constrained to be for real Hilbert spaces. Thus, in this work it is given the definition of the real $L_{2 \mathbb{R}}^{n}[a, b]$ space, and from that function space other Hilbert spaces used by Electrical Engineers will be constructed.

\section{B. Real $L_{2 \mathbb{R}}^{n}[a, b]$ space}

This real Hilbert space is used to describe the voltages and currents in a polyphase system with real-valued functions. The 
elements of $L_{2 \mathbb{R}}^{n}[a, b]$ are the set of all real vector valued functions of dimension $n$, that are square integrable in the finite interval $[a, b]$. Therefore, $\mathbf{X}(t) \in L_{2 \mathbb{R}}^{n}[a, b]$ holds if (8) is true [22],

$$
\begin{array}{r}
\int_{a}^{b} \mathbf{X}(t)^{\top} \mathbf{X}(t) d t<\infty, \\
\mathbf{X}(t)=\left(\begin{array}{lllll}
x_{1}(t) & x_{2}(t) & x_{3}(t) & \ldots & x_{n}(t)
\end{array}\right)^{\top} .
\end{array}
$$

The inner product of this Hilbert space is defined as follows [22],

$$
\langle\mathbf{X}(t), \mathbf{Y}(t)\rangle \triangleq \frac{1}{b-a} \int_{a}^{b} \mathbf{X}(t)^{\top} \mathbf{Y}(t) d t .
$$

Where the vector $\mathbf{Y}(t)$ also belongs to the $L_{2 \mathbb{R}}^{n}[a, b]$ space. From (9), it is obtained the induced norm of $\mathbf{X}(t)$.

$$
\|\mathbf{X}(t)\| \triangleq \sqrt{\frac{1}{b-a} \int_{a}^{b} \mathbf{X}(t)^{\top} \mathbf{X}(t) d t}
$$

With the definitions of the inner product (9) and the norm (10) for $L_{2 \mathbb{R}}^{n}[a, b]$, it is obtained the Cauchy-Schwarz inequality (3). Where this inequality becomes an equality if it is added the term,

$$
\frac{1}{2(b-a)^{2}} \sum_{r=1}^{n} \sum_{s=1}^{n} \int_{a}^{b} \int_{a}^{b}\left(y_{r}(t) x_{s}(\tau)-y_{s}(\tau) x_{r}(t)\right)^{2} d t d \tau .
$$

The term (11) is obtained by adding a correction term to the Cauchy-Schwarz inequality, and following the procedure described in [23, p. 38]. The definition of the term (11) is important because it is a measure of how $\mathbf{X}(t)$ and $\mathbf{Y}(t)$ are not proportional to each other, and also it measures the defect in the Cauchy-Schwarz inequality [23]. Thus, the equality for the $L_{2 \mathbb{R}}^{n}[a, b]$ space is,

$$
\begin{aligned}
& \left(\frac{1}{b-a} \int_{a}^{b} \mathbf{X}(t)^{\top} \mathbf{Y}(t) d t\right)^{2} \\
& +\frac{1}{2(b-a)^{2}} \sum_{r=1}^{n} \sum_{s=1}^{n} \int_{a}^{b} \int_{a}^{b}\left(y_{r}(t) x_{s}(\tau)\right. \\
& \quad-\frac{1}{(b-a)^{2}}\left(\int_{a}^{b} \mathbf{X}(t)^{\top} \mathbf{X}(t) d t\right)\left(\int_{a}^{b} \mathbf{Y}(t)^{\top} \mathbf{Y}(t) d t\right) .
\end{aligned}
$$

Finally, the functions used to form the basis of $L_{2 \mathbb{R}}^{n}[a, b]$ are given in the set $\{\cos (2 \pi f k t)\}_{k=0}^{\infty} \cup\{\sin (2 \pi f k t)\}_{k=0}^{\infty}$. Then, every element of $L_{2 \mathbb{R}}^{n}[a, b]$ is described by the linear combination,

$$
\mathbf{X}(t)=\left(\begin{array}{c}
\sum_{k=0}^{\infty}\left(a_{k 1} \cos (2 \pi f k t)+b_{k 1} \sin (2 \pi f k t)\right) \\
\sum_{k=0}^{\infty}\left(a_{k 2} \cos (2 \pi f k t)+b_{k 2} \sin (2 \pi f k t)\right) \\
\vdots \\
\sum_{k=0}^{\infty}\left(a_{k n} \cos (2 \pi f k t)+b_{k n} \sin (2 \pi f k t)\right)
\end{array}\right) .
$$

Here, $f$ is the fundamental frequency defined by $f=\frac{1}{b-a}$, $k$ is the harmonic number, $a_{0}$ is the constant offset, the variables $a_{k}$ and $b_{k}$ are the amplitudes of the sines and cosines respectively, and since the offset is given in $a_{0}$ then, $b_{0}=0$.

\section{Isomorphism between Hilbert spaces}

The function space described before is written in the time domain, and we would like to also be able to analyze the functions in the frequency domain by obtaining the same results as in the $L_{2 \mathbb{R}}^{n}[a, b]$ space. To accomplish that, it is used the isomorphism between Hilbert spaces. This mathematical concept tells us that two Hilbert spaces are essentially the same if there is a one to one relation between the elements of the Hilbert spaces. The last statement is captured in Theorem 2 .

Theorem 2: Two Hilbert spaces $\mathcal{H}$ and $\widetilde{\mathcal{H}}$, both real or both complex, are isomorphic if and only if they have the same dimension [24].

From Theorem 2, it is constructed the Hilbert space used to represent functions in the frequency domain. For exposition purposes our attention is restricted to the isomorphism of the $L_{2 \mathbb{R}}^{1}[a, b]$ space.

\section{Real $\ell_{2 \mathbb{R}}^{1}$ space}

The real $\ell_{2 \mathbb{R}}^{1}$ space is composed of the real sequences $\left(a_{k}, b_{k}\right)_{k=0}^{\infty}$. From the element in the first row of the linear combination (13), it can be seen that there is a one to one map between the coefficients of the $L_{2 \mathbb{R}}^{1}[a, b]$ space and the sequences that define $\ell_{2 \mathbb{R}}^{1}$. Because of this one to one map, $\ell_{2 \mathbb{R}}^{1}$ is called the coordinate form of $L_{2 \mathbb{R}}^{1}[a, b]$, and these two Hilbert spaces are isomorphic [19]. Thus, to obtain the inner product (9), norm (10) and the term (11) for the $\ell_{2 \mathbb{R}}^{1}$ space, we use the functions belonging to $L_{2 \mathbb{R}}^{1}[a, b]$,

$$
\begin{aligned}
& x(t)=\sum_{k=0}^{\infty}\left(a_{k} \cos (2 \pi f k t)+b_{k} \sin (2 \pi f k t)\right), \\
& y(t)=\sum_{k=0}^{\infty}\left(c_{k} \cos (2 \pi f k t)+d_{k} \sin (2 \pi f k t)\right) .
\end{aligned}
$$

Where the constants $f, k, a_{k}, b_{k}, c_{k}$ and $d_{k}$ have the same meaning as in (13).

Therefore, by using the linear combinations (14) and (15) in the $L_{2 \mathbb{R}}^{1}[a, b]$ definition of the integrals (9)-(11), it is obtained, 


$$
\begin{array}{r}
\frac{1}{b-a} \int_{a}^{b} x(t) y(t) d t=a_{0} c_{0}+\frac{1}{2} \sum_{k=1}^{\infty}\left(a_{k} c_{k}+b_{k} d_{k}\right), \\
\sqrt{\frac{1}{b-a} \int_{a}^{b} x(t)^{2} d t}=\sqrt{a_{0}^{2}+\frac{1}{2} \sum_{k=1}^{\infty}\left(a_{k}^{2}+b_{k}^{2}\right),} \\
\frac{f^{2}}{2} \int_{a}^{b} \int_{a}^{b}(y(t) x(\tau)-y(\tau) x(t))^{2} d t d \tau=Q_{-}+Q_{\sim}, \\
Q_{-}=\frac{1}{2} \sum_{k=1}^{\infty}\left(\left(c_{0} a_{k}-a_{0} c_{k}\right)^{2}+\left(c_{0} b_{k}-a_{0} d_{k}\right)^{2}\right), \\
Q_{\sim}=\frac{1}{8} \sum_{k=1}^{\infty} \sum_{p=1}^{\infty}\left(A^{2}+B^{2}+2 C^{2}\right), \\
A=a_{p} c_{k}-a_{k} c_{p}, \\
B=b_{p} d_{k}-b_{k} d_{p}, \\
C=a_{k} d_{p}-c_{k} b_{p} .
\end{array}
$$

From the equations that define the inner product (16), norm (17) and the term that measures the defect in the CauchySchwarz inequality (18) in the $\ell_{2 \mathbb{R}}^{1}$ space, it can be constructed an equality equivalent to (12) for $\ell_{2 \mathbb{R}}^{1}$. Another piece of information that can be obtain from (18) is that it is composed of a term $Q_{-}$that is just present if there is a constant bias in $x(t)$ and $y(t)$, and a term $Q_{\sim}$ depends on the amplitudes of the sines and cosines describing the functions $x(t)$ and $y(t)$.

The results presented in this section are valid for the $\ell_{2 \mathbb{R}}^{1}$ space, but they can be generalized to the real $\ell_{2 \mathbb{R}}^{n}$ space that is isomorphic to $L_{2 \mathbb{R}}^{n}[a, b]$, following the same steps that were used to derive (16)-(20).

\section{E. Complexification of Hilbert spaces}

Until here, we have presented the results for the real $L_{2 \mathbb{R}}^{n}[a, b]$ and $\ell_{2 \mathbb{R}}^{1}$ spaces, but Electrical Engineers tend to use complex numbers to represent sinusoidal functions in the frequency domain, that they call phasors. Nevertheless, from Theorem 2 it is not possible to obtain a complex Hilbert space, such as it was done before to obtain $\ell_{2 \mathbb{R}}^{1}$. Because it is stated in Theorem 2 that both Hilbert spaces must be complex or real. Thus, we will give the definition of the complexification of a real Hilbert space [25], in order to be able to study the phasorial representation of oscillatory functions.

Definition 1: Suppose that $\mathcal{H}$ is a real Hilbert space. Then, its complexification $\mathcal{H}_{\mathbb{C}}$ is defined as,

$$
\mathcal{H}_{\mathbb{C}} \triangleq\{x+j y \mid x, y \in \mathcal{H}\} .
$$

The addition and multiplication on the complexified space $\mathcal{H}_{\mathbb{C}}$ are defined by (22) and (23), respectively,

$$
\begin{aligned}
& (\hat{x}+j \hat{y})+(\hat{w}+j \hat{z})=(\hat{x}+\hat{w})+j(\hat{y}+\hat{z}), \\
& (a+j b)(\hat{x}+j \hat{y})=(a \hat{x}-b \hat{y})+j(a \hat{y}+b \hat{x}) .
\end{aligned}
$$

Where $\hat{w}, \hat{x}, \hat{y}, \hat{z} \in \mathcal{H}$ and $a, b \in \mathbb{R}$.

Finally, the inner product of the complexified Hilbert space $\mathcal{H}_{\mathbb{C}}$ is defined as [25], $\langle\hat{x}+j \hat{y}, \hat{w}+j \hat{z}\rangle_{\mathcal{H}} \triangleq\langle\hat{x}, \hat{w}\rangle_{\mathcal{H}}+\langle\hat{y}, \hat{z}\rangle_{\mathcal{H}}+j\left(\langle\hat{x}, \hat{z}\rangle_{\mathcal{H}}-\langle\hat{y}, \hat{w}\rangle_{\mathcal{H}}\right)$.

Note that with the definition of the inner product (24), the induced norm in $\mathcal{H}_{\mathbb{C}}$ is given by [25],

$$
\|\hat{x}+j \hat{y}\|_{\mathcal{H}_{\mathbb{C}}}^{2}=\|\hat{x}-j \hat{y}\|_{\mathcal{H}_{\mathbb{C}}}^{2}=\|\hat{x}\|_{\mathcal{H}}^{2}+\|\hat{y}\|_{\mathcal{H}}^{2} .
$$

And the relation between the inner product of $\mathcal{H}$ and $\mathcal{H}_{\mathbb{C}}$ is given by the real part of the inner product of the complexified Hilbert space,

$$
\langle\hat{x}, \hat{y}\rangle_{\mathcal{H}}=\operatorname{Re}\langle\hat{x}, \hat{y}\rangle_{\mathcal{H}_{\mathbb{C}}} .
$$

With Definition 1, we are able to construct the complexification of $L_{2 \mathbb{R}}^{1}[a, b]$ by pairing its basis functions. Thus, the complexified basis functions are given by the set $\{\cos (2 \pi f k t)+$ $j \sin (2 \pi f k t)\}_{k=0}^{\infty}$. Where the complex sum of the sine and cosine is equal to the complex exponential. And as defined in (23) the numbers multiplying the basis in the linear combinations of this complexified space are complex. Therefore, it will be used the complex $L_{2 \mathbb{C}}^{1}[a, b]$ space to study the complexification of $L_{2 \mathbb{R}}^{1}[a, b]$.

\section{F. Complex $L_{2 \mathbb{C}}^{1}[a, b]$ space}

In this complex Hilbert space, a complex function $\mathfrak{x}(t)$ belonging to $L_{2 \mathbb{C}}^{1}[a, b]$ must satisfy the square integrable condition (27) [19], [21],

$$
\int_{a}^{b} \mathfrak{x}(t) \overline{\mathfrak{x}(t)} d t<\infty
$$

Here the inner product is defined as (28) [19], [21], where $\mathfrak{y}(t) \in L_{2 \mathbb{C}}^{1}[a, b]$,

$$
\langle\mathfrak{x}(t), \mathfrak{y}(t)\rangle \triangleq \frac{1}{b-a} \int_{a}^{b} \mathfrak{x}(t) \overline{\mathfrak{y}(t)} d t .
$$

The induced norm by (28) is given as,

$$
\|\mathfrak{x}(t)\| \triangleq \sqrt{\frac{1}{b-a} \int_{a}^{b}|\mathfrak{x}(t)|^{2} d t} .
$$

The term that measures the defect in the Cauchy-Schwarz inequality is given by,

$$
\frac{1}{2(b-a)^{2}} \int_{a}^{b} \int_{a}^{b}|\mathfrak{x}(t) \mathfrak{y}(\tau)-\mathfrak{x}(\tau) \mathfrak{y}(t)|^{2} d t d \tau .
$$

Therefore, the equality for the $L_{2 \mathbb{C}}^{1}[a, b]$ space is given in (31),

$$
\begin{aligned}
& \left|\frac{1}{b-a} \int_{a}^{b} \mathfrak{x}(t) \overline{\mathfrak{y}(t)} d t\right|^{2} \\
& +\frac{1}{2(b-a)^{2}} \int_{a}^{b} \int_{a}^{b}|\mathfrak{x}(t) \mathfrak{y}(\tau)-\mathfrak{x}(\tau) \mathfrak{y}(t)|^{2} d t d \tau \\
& \quad=\frac{1}{(b-a)^{2}}\left(\int_{a}^{b}|\mathfrak{x}(t)|^{2} d t\right)\left(\int_{a}^{b}|\mathfrak{y}(t)|^{2} d t\right)
\end{aligned}
$$


All elements of the $L_{2 \mathbb{C}}^{1}[a, b]$ space can be written as the linear combination,

$$
\mathfrak{x}(t)=\sum_{k=0}^{\infty} \mathbf{X}_{k} e^{j 2 \pi f k t}
$$

Where $f$ is the fundamental frequency, $k$ is the harmonic number and $\mathbf{X}_{k}$ is a complex number.

In order to achieve the same previous results (16)-(20), in the complexification of $L_{2 \mathbb{R}}^{1}[a, b]$, we need to properly select the values of $\mathbf{X}_{k}$. This is done by following the complexification of real functions used by Electrical Engineers,

$$
\sqrt{a_{k}^{2}+b_{k}^{2}} \cos (2 \pi f k t+\phi) \mapsto\left|\mathbf{X}_{k}\right| e^{j \phi} e^{j 2 \pi f k t}
$$

From the map (33) done by Electrical Engineers, the relation between the complex numbers $\mathbf{X}_{k}$ and the real numbers $a_{k}, b_{k}$ that denote the amplitudes of the sines and cosines in $L_{2 \mathbb{R}}^{1}[a, b]$ is given by,

$$
\begin{array}{r}
\mathbf{X}_{0}=a_{0}, \\
\mathbf{X}_{k}=\frac{1}{\sqrt{2}}\left(a_{k}-j b_{k}\right) \quad k=1, \ldots, \infty
\end{array}
$$

Then, with the relations (34)-(35), it is obtained the equality of the norms (17) and (29),

$$
\sqrt{\frac{1}{b-a} \int_{a}^{b} x(t)^{2} d t}=\sqrt{\frac{1}{b-a} \int_{a}^{b}|\mathfrak{x}(t)|^{2} d t}
$$

The equality of the inner products (16) and (28),

$$
\frac{1}{b-a} \int_{a}^{b} x(t) y(t) d t=\operatorname{Re}\left(\frac{1}{b-a} \int_{a}^{b} \mathfrak{x}(t) \overline{\mathfrak{y}(t)} d t\right) .
$$

And the equality of the terms that measure the defect in the Cauchy-Schwarz inequality, given in (18), (28) and (30),

$$
\begin{aligned}
& \frac{1}{2(b-a)^{2}} \int_{a}^{b} \int_{a}^{b}(y(t) x(\tau)-y(\tau) x(t))^{2} d t d \tau \\
& =\frac{1}{2(b-a)^{2}} \int_{a}^{b} \int_{a}^{b}|\mathfrak{x}(t) \mathfrak{y}(\tau)-\mathfrak{x}(\tau) \mathfrak{y}(t)|^{2} d t d \tau \\
& \quad+\left(\operatorname{Im}\left(\frac{1}{b-a} \int_{a}^{b} \mathfrak{x}(t) \overline{\mathfrak{y}(t)} d t\right)\right)^{2}
\end{aligned}
$$

Here, it was constructed the complexification of $L_{2 \mathbb{R}}^{1}[a, b]$. That is composed of complex functions in the time domain, but this is not the concept of phasors used by Electrical Engineers. Therefore, to construct a complex Hilbert space that allows us to study the phasorial representation of the functions, we will use Theorem 2 to construct the complex $\ell_{2 \mathbb{C}}^{1}$ space from $L_{2 \mathbb{C}}^{1}[a, b]$.

\section{G. Complex $\ell_{2 \mathbb{C}}^{1}$ space}

The last Hilbert space that will be described here is the $\ell_{2 \mathbb{C}}^{1}$ space, that is the coordinate form of the $L_{2 \mathbb{C}}^{1}[a, b]$ space [19]. The elements belonging to this space are called phasors by Electrical Engineers. This space is composed with the complex sequences $\left(\mathbf{X}_{k}\right)_{k=0}^{\infty}$, that satisfy (39) [19],

$$
\sum_{k=0}^{\infty}\left|\mathbf{X}_{k}\right|^{2}<\infty
$$

To obtain the relations between $L_{2 \mathbb{C}}^{1}[a, b]$ and $\ell_{2 \mathbb{C}}^{1}$, we use the complex functions,

$$
\begin{aligned}
& \mathfrak{x}(t)=\sum_{k=0}^{\infty} \mathbf{X}_{k} e^{j 2 \pi f k t}, \\
& \mathfrak{y}(t)=\sum_{k=0}^{\infty} \mathbf{Y}_{k} e^{j 2 \pi f k t} .
\end{aligned}
$$

Thus, by using the linear combinations (40) and (41) in the $L_{2 \mathbb{C}}^{1}[a, b]$ definition of the integrals (28)-(30), it is obtained the relation between inner products,

$$
\frac{1}{b-a} \int_{a}^{b} \mathfrak{x}(t) \overline{\mathfrak{y}(t)} d t=\sum_{k=0}^{\infty} \mathbf{X}_{k} \overline{\mathbf{Y}}_{k}
$$

The equality of the norms,

$$
\sqrt{\frac{1}{b-a} \int_{a}^{b}|\mathfrak{x}(t)|^{2} d t}=\sqrt{\sum_{k=0}^{\infty}\left|\mathbf{X}_{k}\right|^{2}} .
$$

And the relation between the terms that measure the defect in the Cauchy-Schawz inequality,

$$
\begin{aligned}
& \frac{1}{2(b-a)^{2}} \int_{a}^{b} \int_{a}^{b}|\mathfrak{x}(t) \mathfrak{y}(\tau)-\mathfrak{x}(\tau) \mathfrak{y}(t)|^{2} d t d \tau \\
& =\frac{1}{2} \sum_{k=0}^{\infty} \sum_{p=0}^{\infty}\left|\mathbf{X}_{k} \mathbf{Y}_{p}-\mathbf{X}_{p} \mathbf{Y}_{k}\right|^{2}
\end{aligned}
$$

From the relations (42)-(44), it is represented the equation (31) in the $\ell_{2 \mathbb{C}}^{1}$ space. Where this equation is called the complex Lagrange's identity (45) [23],

$$
\begin{aligned}
&\left|\sum_{k=0}^{\infty} \mathbf{X}_{k} \overline{\mathbf{Y}}_{k}\right|^{2}+\frac{1}{2} \sum_{k=0}^{\infty} \sum_{p=0}^{\infty}\left|\mathbf{X}_{k} \mathbf{Y}_{p}-\mathbf{X}_{p} \mathbf{Y}_{k}\right|^{2} \\
&=\sum_{k=0}^{\infty}\left|\mathbf{X}_{k}\right|^{2} \sum_{k=0}^{\infty}\left|\mathbf{Y}_{k}\right|^{2}
\end{aligned}
$$

When the Lagrange's identity (45) is composed just by $\mathbf{X}_{1}$ and $\mathbf{Y}_{1}$, it is called the Fibonnaci-Brahmagupta equation [23].

In this section were given four different Hilbert spaces that provide different ways to represent functions. And by properly selecting the relations of the coefficients in the complex Hilbert spaces achieve the same results, even if the Hilbert spaces are not isomorphic. The theory given here serves as the basis to derive the generalized definition of the reactive power. 


\section{THE GENERALIZED REACTIVE POWER}

In Section II-A, it was given the Least effort problem defined in a real Hilbert space $\mathcal{H}$. Here, it will be used that problem with one constrain to obtain the generalized reactive power $Q_{\mathcal{H}}$ in $\mathcal{H}$. Then, this general result for real Hilbert spaces will be used to obtain the reactive power of a multiphase system described by the $L_{2 \mathbb{R}}^{n}[0, T]$ space.

\section{A. Derivation of the generalized reactive power using the Least-effort problem}

The Least effort problem used in this work is the generalization of the minimization problem approach that was developed in the Institute of Electrical Circuit Theory and Engineering in 1985 [6]. Where this abstract variational problem is looking for the current that achieves the minimum norm defined in a real Hilbert space $\mathcal{H}$ and the same average electrical power transfer. Since the average transmission of power is defined by an inner product in $\mathcal{H}$, then this is the constrain in the problem. The reason why this problem was defined in a real Hilbert spaces is because we want to have the notion of the angle (2) between two functions in $\mathcal{H}$. Thus, the Least effort problem is written as follows,

$$
\begin{array}{ll}
\underset{\hat{i} \in \mathcal{H}}{\operatorname{minimize}} & \|\hat{i}\|^{2}, \\
\text { subject to } & P_{\mathcal{H}}=\langle\hat{i}, \hat{v}\rangle .
\end{array}
$$

Here, $\hat{i}$ and $\hat{v}$ are the current and voltage of a circuit element represented in $\mathcal{H}$, and $P_{\mathcal{H}}$ is the average electrical power transfer of that circuit element, represented by the inner product in $\mathcal{H}$.

In order to solve for the minimum norm current $\hat{i}_{0}$, the results presented in Section II-A are used. Firstly, we need to look for the basis of the subspace $\mathcal{M}$. By the definition of the constrain in (46), this basis is defined by the vector $\hat{v}$. Then, by Theorem $1, \hat{i}_{0}$ is a linear combination of the $\mathcal{M}$ basis, and it is written as,

$$
\hat{i}_{0}=\beta_{\mathcal{H}} \hat{v}
$$

To obtain $\beta_{\mathcal{H}}$, we need to substitude (47) in the constrain defined in (46). This substitution constructs the Gram equation defined in (7), and with that we can solve for $\beta_{\mathcal{H}}$,

$$
\beta_{\mathcal{H}}=\frac{P_{\mathcal{H}}}{\langle\hat{v}, \hat{v}\rangle}=\frac{P_{\mathcal{H}}}{\|\hat{v}\|^{2}} .
$$

Here, $\beta_{\mathcal{H}}$ will be called the generalized Fryze conductace. Where this conductance defines the relation between $\hat{i}_{0}$ and $\hat{v}$,

$$
\hat{i}_{0}=\frac{P_{\mathcal{H}}}{\|\hat{v}\|^{2}} \hat{v} .
$$

Equation (49) solves for the minimum norm current that achieves the same average power transfer described in (46). Where the only passive elements that can achieve (49) are linear resistors. Other passive circuit elements must have compensation circuits to attain (49).

Once that we solve the minimization problem (46). It is studied the geometrical properties of the real Hilbert space $\mathcal{H}$ to obtain the definition of the generalized reactive power $Q_{\mathcal{H}}$. To do that, we rely on the proof of Theorem 1, that is briefly described in Section II-A, and for this problem it is depicted in Fig. 2.

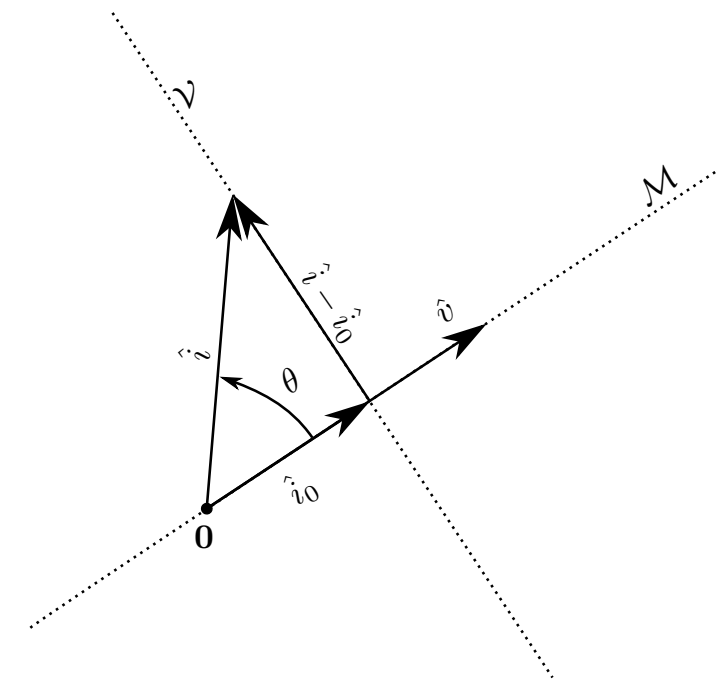

Fig. 2. Geometrical interpretation of the power triangle.

Here, $\theta$ is the notion of an angle between the two functions $\hat{i}, \hat{v} \in \mathcal{H}$, and the hyperplane $\mathcal{V}$ is the intersection of the constrains in (46). Since in (46) there is just one constrain, the hyperplane $\mathcal{V}$ is defined just by $P_{\mathcal{H}}=\langle\hat{i}, \hat{v}\rangle$. From Fig. 2, we derive the relations between the norms of the minimizing current $\hat{i}_{0}$, orthogonal current $\hat{i}-\hat{i}_{0}$ and the current of the circuit element $\hat{i}$, where these relations are given as (50)-(51),

$$
\begin{aligned}
\left\|\hat{i}_{0}\right\| & =\|\hat{i}\| \cos (\theta), \\
\left\|\hat{i}-\hat{i}_{0}\right\| & =\|\hat{i}\| \sin (\theta) .
\end{aligned}
$$

Where $\cos (\theta)$ is the generalized power factor.

Since the minimizing current $\hat{i}_{0}$ and the orthogonal current $\hat{i}-\hat{i}_{0}$ are orthogonal to each other, we can add them in quadrature and obtain the norm of $\hat{i}(52)$,

$$
\|\hat{i}\|^{2}=\left\|\hat{i}_{0}\right\|^{2}+\left\|\hat{i}-\hat{i}_{0}\right\|^{2}
$$

Equation (52) tells us that the orthogonal current $\hat{i}-\hat{i}_{0}$ affects the ampacity of the electrical network the circuit element is connected to. Thus, the norm of this vector shall be as low as possible. The minimization of $\hat{i}-\hat{i}_{0}$ can just be done if we know its direction and norm in $\mathcal{H}$. Therefore, a current with opposite direction and the same norm shall be applied to compensate for $\hat{i}-\hat{i}_{0}$, and attain $\hat{i}_{0}$.

Another way to write the quadrature equation (52), is by multiplying by the squared norm of the circuit element voltage $\hat{v}$,

$$
\|\hat{v}\|^{2}\|\hat{i}\|^{2}=\|\hat{v}\|^{2}\left\|\hat{i}_{0}\right\|^{2}+\|\hat{v}\|^{2}\left\|\hat{i}-\hat{i}_{0}\right\|^{2} .
$$

The equation (53) is the relation describing the generalized power triangle. The terms in (53) are rewritten by substituting equations (50)-(51) in (53), 


$$
\|\hat{v}\|^{2}\|\hat{i}\|^{2}=\|\hat{v}\|^{2}\|\hat{i}\|^{2} \cos ^{2}(\theta)+\|\hat{v}\|^{2}\|\hat{i}\|^{2} \sin ^{2}(\theta) .
$$

The terms in (54) will be called the generalized apparent $S_{\mathcal{H}}$ (55), active $P_{\mathcal{H}}$ (56) and reactive $Q_{\mathcal{H}}$ (57) powers.

$$
\begin{array}{r}
S_{\mathcal{H}}=\|\hat{v}\|\|\hat{i}\|, \\
P_{\mathcal{H}}=\|\hat{v}\|\|\hat{i}\| \cos (\theta), \\
Q_{\mathcal{H}}=\|\hat{v}\|\|\hat{i}\| \sin (\theta) .
\end{array}
$$

If we do not add $Q_{\mathcal{H}}$ (57) to (54), take the square root at both sides and recall (2). It is obtained the Cauchy-Schwarz inequality,

$$
\langle\hat{i}, \hat{v}\rangle \leq\|\hat{v}\|\|\hat{i}\| .
$$

Thus, the generalized power triangle is a property of the real Hilbert space used to represent the voltages and currents, and it gives another approach to attain the minimizing current $\hat{i}_{0}$, if $\hat{i}-\hat{i}_{0}$ is known.

\section{B. Reactive power of multiphase systems}

The result derived in Section III-A will be used here to obtain the reactive power for a multiphase system. A graphical representation of how the voltages and currents are measured in these systems is depicted in Fig. 3.

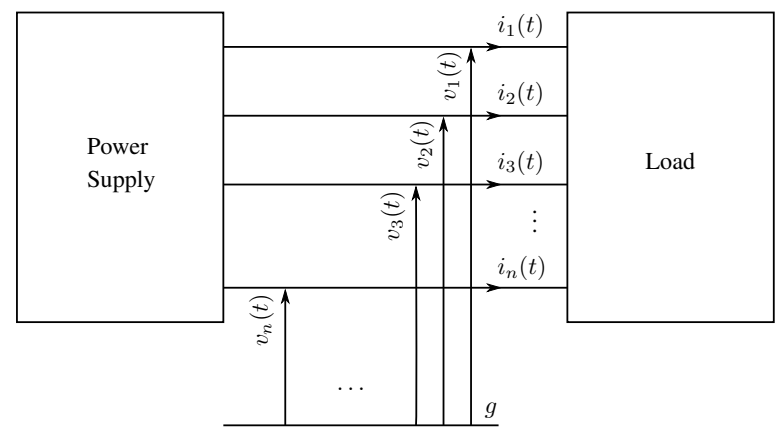

Fig. 3. Interconnection between a load and a power supply.

Where $v_{k}(t)$ is the $k$ th function representing the voltage with respect to ground, and $i_{k}(t)$ is the $k$ th the function that captures line current. Afterwards, we gather together these voltages and currents into the real-valued vector functions (59)-(60),

$$
\begin{gathered}
\mathbf{V}(t)=\left(\begin{array}{lllll}
v_{1}(t) & v_{2}(t) & v_{3}(t) & \ldots & v_{n}(t)
\end{array}\right)^{\top} \\
\mathbf{I}(t)=\left(\begin{array}{lllll}
i_{1}(t) & i_{2}(t) & i_{3}(t) & \ldots & i_{n}(t)
\end{array}\right)^{\top} .
\end{gathered}
$$

From the definition of how the voltages and currents are represented (59)-(60), the real $L_{2 \mathbb{R}}^{n}[0, T]$ space is used to express the Least effort problem (46). Using the definitions for the inner product (9) and norm (10) for $L_{2 \mathbb{R}}^{n}[0, T]$. The problem is defined as,

$$
\begin{array}{ll}
\underset{\mathbf{I}(t) \in L_{2}^{n}[0, T]}{\operatorname{minimize}} & \frac{1}{T} \int_{0}^{T} \mathbf{I}(t)^{\top} \mathbf{I}(t) d t, \\
\text { subject to } & P=\frac{1}{T} \int_{0}^{T} \mathbf{I}(t)^{\top} \mathbf{V}(t) d t .
\end{array}
$$

Here, $T$ is the period of the fundamental frequency, $\mathbf{I}(t)$ and $\mathbf{V}(t)$ represent the currents and voltages of the load depicted in Fig. 3, where the average electrical power transfer for $L_{2 \mathbb{R}}^{n}[0, T]$ is represented as $P$.

The current $\mathbf{I}(t)_{0}$ that minimizes (61) is obtained by following the procedure used to get (49). The minimum current for $L_{2 \mathbb{R}}^{n}[0, T]$ is given by,

$$
\mathbf{I}(t)_{0}=\frac{P}{\frac{1}{T} \int_{0}^{T} \mathbf{V}(t)^{\top} \mathbf{V}(t) d t} \mathbf{V}(t) .
$$

Once the minimum current $\mathbf{I}(t)_{0}$ is obtained, we use the generalized power triangle depicted in Fig. 2 to obtain the apparent power $S$ (63) by using (55) and the norm definition in (10),

$$
S=\sqrt{\frac{1}{T} \int_{0}^{T} \mathbf{V}(t)^{\top} \mathbf{V}(t) d t} \sqrt{\frac{1}{T} \int_{0}^{T} \mathbf{I}(t)^{\top} \mathbf{I}(t) d t .}
$$

The active $P$ power (64) is obtained by using the general definition of the active power (56) and the definition of the inner product in $L_{2 \mathbb{R}}^{n}[0, T](9)$,

$$
P=\frac{1}{T} \int_{0}^{T} \mathbf{I}(t)^{\top} \mathbf{V}(t) d t
$$

For the definition of the reactive power $Q(65)$ in $L_{2 \mathbb{R}}^{n}[0, T]$, we need to use the result stating that the apparent (63) and active (64) powers construct the Cauchy-Schwarz inequality (58). Then, by using equality (12), we can see that the term related to $Q$ is given by (11). Thus, by rewriting (11) using $\mathbf{V}(t)$ and $\mathbf{I}(t)$, it is obtained,

$$
Q=\sqrt{\frac{1}{2 T^{2}} \sum_{r=1}^{n} \sum_{s=1}^{n} \int_{0}^{T} \int_{0}^{T}\left(v_{r}(t) i_{s}(\tau)-v_{s}(\tau) i_{r}(t)\right)^{2} d t d \tau} .
$$

The "powers" defined here (63)-(65) satisfy the equation $S^{2}=P^{2}+Q^{2}$, and describe the power triangle in $L_{2 \mathbb{R}}^{n}[0, T]$. Thus, these "powers" are just a geometrical property of the $L_{2 \mathbb{R}}^{n}[0, T]$ space.

Finally, the same methodology followed in this section can be used to derive the value describing the reactive power in another real Hilbert space. And if we want to use a complex Hilbert space, we can do it by the proper complexification of a real Hilbert space as defined in Section II-E.

\section{COMPARISON WITH OTHER POWER THEORIES}

The derivation of the generalized reactive power $Q_{\mathcal{H}}$ given in Section III will be used here to prove that the Classical power theory, Fryze's power theory and the Instantaneous power theory capture just particular cases of the whole reactive power problem. Also, it will be explained why the Budeanu's power theory does not capture the power phenomena. 


\section{A. Classical power theory}

This theory was the first one used to describe the reactive power. The idea of this theory is to threat the power of a sinusoidal signal as an average value of over period of the measured signal $T$. Since the measured quantities are sinusoidal and the models used are Linear Time Invariant (LTI), we use phasors to represent the sinusoidal voltage (66) and current (67),

$$
\begin{aligned}
\mathbf{V}_{c} & =\frac{1}{\sqrt{2}}\left(v_{1 C}-j v_{1 S}\right), \\
\mathbf{I}_{c} & =\frac{1}{\sqrt{2}}\left(i_{1 C}-j i_{1 S}\right) .
\end{aligned}
$$

Here, $\mathbf{V}_{c}$ and $\mathbf{I}_{c}$ represent a sinusoidal voltage and current expressed in the $\ell_{2 \mathbb{C}}^{1}$ space. According to the complexification of the sinusoidal amplitudes (35) the values $v_{1 C}, v_{1 S}, i_{1 C}$ and $i_{1 S}$ are the cosine and sine amplitudes of the sinusoidal signal representing the voltages and currents in a real Hilbert space.

With the complex quantities defined in (66)-(67), it is obtained the classical apparent power $\mathbf{S}_{c}$,

$$
\mathbf{S}_{c}=\mathbf{V}_{c} \overline{\mathbf{I}}_{c}=\underbrace{\frac{1}{2}\left(v_{1 C} i_{1 C}+v_{1 S} i_{1 S}\right)}_{P_{c}}+j \underbrace{\frac{1}{2}\left(v_{1 C} i_{1 S}-v_{1 S} i_{1 C}\right)}_{Q_{c}} .
$$

Where $P_{c}$ is the classical active power and $Q_{c}$ is the classical reactive power. By taking the complex modulus to (68), we obtain the following equality,

$$
\left|\mathbf{S}_{c}\right|^{2}=P_{c}^{2}+Q_{c}^{2} .
$$

Where the equality (69) represents the power triangle in the Classical power theory. Since $\mathbf{V}_{c}, \mathbf{I}_{c} \in \ell_{2 \mathbb{C}}^{1}$ the equation (69) is the Fibonnaci-Brahmagupta equation or the complex Lagrange's identity just with the fundamental component (45). Thus, the Classical power theory is solving for the Least-effort problem under sinusoidal conditions in the $\ell_{2 \mathbb{C}}^{1}$ space.

\section{B. Fryze's power theory}

Fryze developed his theory using the Cauchy-Schwarz inequality [3]. Where he obtained the particular case of the generalized Fryze conductance (48) for the $L_{2 \mathbb{R}}^{1}[0, T]$ space, but he did not give a physical interpretation of the problem. To obtain Fryze's results, we just need to solve the Leasteffort problem in the $L_{2 \mathbb{R}}^{1}[0, T]$ space. Where we obtain the following expression for the Fryze's current $i_{1}(t)_{0}$,

$$
i_{1}(t)_{0}=\frac{P_{F}}{\left\|v_{1}(t)\right\|^{2}} v_{1}(t)=\frac{\frac{1}{T} \int_{0}^{T} v_{1}(t) i_{1}(t) d t}{\frac{1}{T} \int_{0}^{T} v_{1}(t)^{2} d t} v_{1}(t) .
$$

Here, $i_{1}(t)$ and $v_{1}(t)$ are the current and voltage of the circuit element under study, and the average electrical power transfer is given by $P_{F}$.

Therefore, the Fryze's current (70) is just a particular case of the solution for the Least-effort problem (46), when the space used to represent the voltages and currents of a circuit is the $L_{2 \mathbb{R}}^{1}[0, T]$ space.

\section{Instantaneous power theory}

This power theory is used to deal with rapidly changing loads, and it is based on the Clarke and Park transformations of the voltages and currents in a balanced three phase system [4], [13]. To obtain the relation between this power theory and the Least-effort problem, we will use the fundamental component of $L_{2 \mathbb{R}}^{3}[0, T]$ space basis (13), to express the voltages (71) and currents (72),

$$
\begin{gathered}
\left(\begin{array}{l}
v_{1}(t) \\
v_{2}(t) \\
v_{3}(t)
\end{array}\right)=\left(\begin{array}{c}
\sqrt{2} V_{\text {ins }} \cos (\omega t) \\
\sqrt{2} V_{\text {ins }} \cos \left(\omega t-\frac{2}{3} \pi\right) \\
\sqrt{2} V_{\text {ins }} \cos \left(\omega t+\frac{2}{3} \pi\right)
\end{array}\right), \\
\left(\begin{array}{c}
i_{1}(t) \\
i_{2}(t) \\
i_{3}(t)
\end{array}\right)=\left(\begin{array}{c}
\sqrt{2} I_{\text {ins }} \cos \left(\omega t-\theta_{i}\right) \\
\sqrt{2} I_{\text {ins }} \cos \left(\omega t-\frac{2}{3} \pi-\theta_{i}\right) \\
\sqrt{2} I_{\text {ins }} \cos \left(\omega t+\frac{2}{3} \pi-\theta_{i}\right)
\end{array}\right) .
\end{gathered}
$$

Here $v_{1}(t), v_{2}(t), v_{3}(t), i_{1}(t), i_{2}(t), i_{3}(t)$ are the phase voltages and line currents, respectively. The angular frequency is given by $\omega, \theta_{i}$ is the phase difference between the voltages and currents, and the RMS values of the voltages and currents with respect to ground are given by $V_{\text {ins }}$ and $I_{\text {ins }}$.

Since the voltages (71) and currents (72) were assumed to be sinusoidal and balanced, they describe circles in a plane perpendicular to the vector $(1,1,1)^{\top}$. Thus, it is more convenient to do the whole computations on that plane.

1) The $p-q$ theory: This instantaneous power theory uses a fixed reference frame in the plane perpendicular to $(1,1,1)^{\top}$, where the voltages (71) and currents (72) are transformed using the power invariant Clarke transformation to,

$$
\begin{array}{r}
\mathbf{V}_{\alpha \beta}(t)=\left(\begin{array}{l}
v_{\alpha}(t) \\
v_{\beta}(t)
\end{array}\right)=\left(\begin{array}{c}
\sqrt{3} V_{\text {ins }} \cos (\omega t) \\
\sqrt{3} V_{\text {ins }} \sin (\omega t)
\end{array}\right), \\
\mathbf{I}_{\alpha \beta}(t)=\left(\begin{array}{l}
i_{\alpha}(t) \\
i_{\beta}(t)
\end{array}\right)=\left(\begin{array}{c}
\sqrt{3} I_{\text {ins }} \cos \left(\omega t-\theta_{i}\right) \\
\sqrt{3} I_{\text {ins }} \sin \left(\omega t-\theta_{i}\right)
\end{array}\right) .
\end{array}
$$

The instantaneous active and reactive powers are defined as,

$$
\begin{gathered}
p_{\alpha \beta}=\mathbf{V}_{\alpha \beta}(t)^{\top} \mathbf{I}_{\alpha \beta}(t)=v_{\alpha}(t) i_{\alpha}(t)+v_{\beta}(t) i_{\beta}(t), \\
q_{\alpha \beta}=\mathbf{V}_{\alpha \beta}(t) \times \mathbf{I}_{\alpha \beta}(t)=v_{\alpha}(t) i_{\beta}(t)-v_{\beta}(t) i_{\alpha}(t) .
\end{gathered}
$$

Using the definition of the voltages (73) and currents (73) in (75)-(76) we obtain,

$$
\begin{gathered}
p_{\alpha \beta}=3 V_{\text {ins }} I_{\text {ins }} \cos \left(\theta_{i}\right), \\
q_{\alpha \beta}=-3 V_{\text {ins }} I_{\text {ins }} \sin \left(\theta_{i}\right) .
\end{gathered}
$$

The results obtained in (77)-(78) are just like the one presented for the generalized power triangle (56)-(57). Where the difference is in the sign of the instantaneous power theory (78), but the absolute value of (57) represents the scaled distance to the minimum norm current for a three phase system under balanced conditions. Thus, for the balanced sinusoidal operation condition [5], the $p-q$ theory is solving for the Least-effort problem in the $L_{2 \mathbb{R}}^{3}[0, T]$ space. 
2) The synchronous reference frame theory: This is a modification of the $p-q$ theory. It is based on a transformation that is moving along the voltage vector (71). That reference frame transformation is done with the Park transformation [13]. The voltages (71) and currents (72) in this new basis are given by,

$$
\begin{array}{r}
\mathbf{V}_{d q}(t)=\left(\begin{array}{c}
v_{d} \\
v_{q}
\end{array}\right)=\left(\begin{array}{c}
\sqrt{3} V_{\text {ins }} \\
0
\end{array}\right), \\
\mathbf{I}_{d q}(t)=\left(\begin{array}{c}
i_{d} \\
i_{q}
\end{array}\right)=\left(\begin{array}{c}
\sqrt{3} I_{\text {ins }} \cos \left(\theta_{i}\right) \\
-\sqrt{3} I_{\text {ins }} \sin \left(\theta_{i}\right)
\end{array}\right) .
\end{array}
$$

The instantaneous active and reactive power are defines as,

$$
\begin{gathered}
p_{S R F}=\mathbf{V}_{d q}(t)^{\top} \mathbf{I}_{d q}(t)=v_{d} i_{d}+v_{q} i_{q}, \\
q_{S R F}=\mathbf{V}_{d q}(t) \times \mathbf{I}_{d q}(t)=v_{d} i_{q}-v_{q} i_{d} .
\end{gathered}
$$

Now, substituting the $d-q$ voltages (79) and currents (80) into (81) and (82), the following results are obtained,

$$
\begin{gathered}
p_{S R F}=3 V_{i n s} I_{i n s} \cos \left(\theta_{i}\right), \\
q_{S R F}=-3 V_{\text {ins }} I_{i n s} \sin \left(\theta_{i}\right) .
\end{gathered}
$$

Just as it happened in the $p-q$ theory, the synchronous reference frame theory is solving for the Least-effort problem assuming that the system is balanced and three phase.

\section{Budeanu's power theory}

This theory was the first attempt to explain the phenomena described by C. P. Steinmetz [1] in 1892. It uses the $\ell_{2 \mathbb{C}}^{1}$ space to define the single phase voltages and currents under nonsinusoidal conditions. Where Budeanu defined the active $P_{B}$, reactive $Q_{B}$, apparent $S_{B}$, and distortion $D_{B}$ powers [2]. The definition of these powers are given in (85)-(88),

$$
\begin{array}{r}
P_{B} \triangleq \sum_{k=1}^{\infty}\left|\mathbf{V}_{B k}\right|\left|\mathbf{I}_{B k}\right| \cos \left(\theta_{v k}-\theta_{i k}\right), \\
Q_{B} \triangleq \sum_{k=1}^{\infty}\left|\mathbf{V}_{B k}\right|\left|\mathbf{I}_{B k}\right| \sin \left(\theta_{v k}-\theta_{i k}\right), \\
S_{B} \triangleq \sqrt{\sum_{k=1}^{\infty}\left|\mathbf{V}_{B k}\right|^{2} \sum_{k=1}^{\infty}\left|\mathbf{I}_{B k}\right|^{2},} \\
D_{B} \triangleq \sqrt{S_{B}^{2}-P_{B}^{2}-Q_{B}^{2}} .
\end{array}
$$

Here, $k$ is the harmonic number, $\left|\mathbf{V}_{B k}\right|$ and $\left|\mathbf{I}_{B k}\right|$ are the complex norms of each voltage and current harmonics, $\phi_{v_{k}}$ is the phase angle of the voltage of the $k$ th harmonic, $\phi_{i_{k}}$ is the phase angle of the current of the $k$ th harmonic.

To understand the meaning of the powers defined by Budeanu, we need to express the phasors representing the voltages and currents into its real and imaginary components,

$$
\begin{array}{r}
\mathbf{V}_{B k}=\left|\mathbf{V}_{B k}\right| \cos \left(\theta_{v k}\right)+j\left|\mathbf{V}_{B k}\right| \sin \left(\theta_{v k}\right) \\
\mathbf{I}_{B k}=\left|\mathbf{I}_{B k}\right| \cos \left(\theta_{i k}\right)+j\left|\mathbf{I}_{B k}\right| \sin \left(\theta_{i k}\right)
\end{array}
$$

Now, using the inner product defined for the $\ell_{2 \mathbb{C}}^{1}$ space (42), we obtain the following relation for the active and reactive powers defined by Budeanu,

$$
\sum_{k=1}^{\infty} \mathbf{V}_{B k} \overline{\mathbf{I}}_{B k}=P_{B}+j Q_{B}
$$

From (26) and (38) we know that the active power defined by Budeanu $P_{B}$ characterizes the average power transmission, and the reactive power $Q_{B}$ is part of the term that measures the non proportionality of the voltages and currents. In fact, $Q_{B}$ defines the defect that is compensated by linear passive components [2].

Since $P_{B}$ and $Q_{B}$ are related with the inner product in the $\ell_{2 \mathbb{C}}^{1}$ space (91), and the apparent power $S_{B}$ is the multiplication of the voltage and current norms in the $\ell_{2 \mathbb{C}}^{1}$ space (87). The distortion power defined by Budeanu (88) is defined according to the complex Lagrange's identity (45) as follows,

$$
D_{B}=\sqrt{\frac{1}{2} \sum_{k=1}^{\infty} \sum_{p=1}^{\infty}\left|\mathbf{V}_{B p} \mathbf{I}_{B k}-\mathbf{V}_{B k} \mathbf{I}_{B p}\right|^{2}} .
$$

Budeanu's power theory uses the complex Lagrange's identity to define the powers to be compensated $Q_{B}$ and $D_{B}$. The problem with this theory is that the direction in $\ell_{2 \mathbb{C}}^{1}$ of the current to be compensated is not defined. Thus, we know from the complex Lagrange's identity the norm of the current that needs to be compensated but we do not know which current. Therefore, it is an incomplete characterization of the Least effort problem in the $\ell_{2 \mathbb{C}}^{1}$ space.

\section{Relation BetweEn the TELLEgEN'S THEOREM AND THE POWER THEORY}

One of the most powerful theorems in network theory is the one proved by B. Tellegen in 1952 [26]. This theorem states that the sum of the product of the voltage and current in every branch is zero. Where the only conditions imposed to the network is that it must follow the Kirchhoff voltage and current laws. The interpretation of this theorem is the one of the conservation of energy in a general network.

In [26], B. Tellegen proved that his theorem also holds for the phasorial representation of sinusoidal voltages and currents. Where he used the inner product in the $\ell_{2 \mathbb{C}}^{1}$ space to prove that the complex "powers" of a load and a power supply are equal.

Here, we will demostrate that the imaginary part of the complex "powers" is in fact not a power, but rather a measure of a defect in the optimality condition for the generalized reactive power problem (46). To do this, it will be used the circuit depicted in Fig. 4, where the voltage $\mathfrak{v}(t)$ and current $\mathfrak{i}(t)$ are represented in the complex $L_{2 \mathbb{C}}^{1}[0, T]$ space, that is isomorphic to the $\ell_{2 \mathbb{C}}^{1}$ space used by $\mathrm{B}$. Tellegen.

As depicted in Fig. 4, the circuit has just a power supply and a circuit load. Here we will follow B. Tellegen's approach by studying just the inner product in $L_{2 \mathbb{C}}^{1}[0, T]$ of the voltage and current for the circuit load. That calculation is given in, 


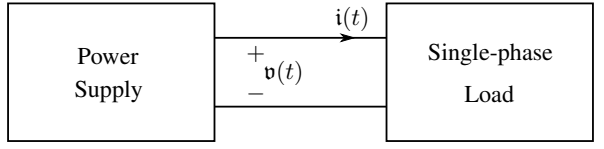

Fig. 4. Single-phase circuit represented in the $L_{2 \mathbb{C}}^{1}[0, T]$ space.

$$
\frac{1}{T} \int_{0}^{T} \mathfrak{v}(t) \overline{\mathfrak{i}(t)} d t
$$

Since (93) is the inner product of $L_{2 \mathbb{C}}^{1}[0, T]$ that is the complexification of $L_{2 \mathbb{R}}^{1}[0, T]$, we can not say that (93) is power because $L_{2 \mathbb{C}}^{1}[0, T]$ and $L_{2 \mathbb{R}}^{1}[0, T]$ are not isomorphic [25]. Therefore, just the real part of (93) has the meaning of power, according to (26) and (37), and the imaginary part of (93) is a part of the measure of a defect in the optimality condition for the generalized reactive power problem (46), according to (38). Thus, Tellegen's theorem does not capture the reactive power problem in the complex $L_{2 \mathbb{C}}^{1}[0, T]$ or $\ell_{2 \mathbb{C}}^{1}$ spaces, unless the voltages and currents are purely sinusoidal, that is the Classical power theory case (Section IV-A).

As a consequence of the explanation given above, the reactive power is not conserved in the sense of Tellegen, unless it is the purely sinusoidal case. This happens because the measures of the defect in the Cauchy-Schwarz inequality given in (44) are zero in the purely sinusoidal case, thus the only term left in the right hand side of (38) is the square of imaginary part of (93).

\section{Circuit EXAMPle}

In previous articles, the authors have described how harmonics and unbalance in $\mathrm{AC}$ circuits produce reactive power [9], [17]. And it is commonly accepted that reactive power does not exist in DC circuits. Thus, using the definition of the generalized reactive power given in Section III, it will be demonstrated that it is possible to have reactive power in a switched DC circuit.

\section{A. Buck converter}

The Buck converter is a switched DC-DC converter that reduces the output voltage with respect to its input voltage [27]. The topology of this converter is depicted in Fig. 5.

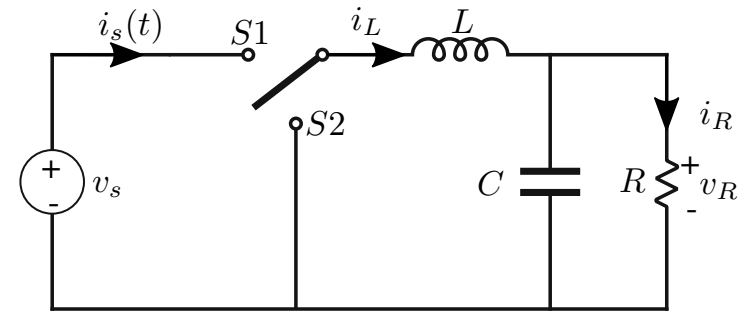

Fig. 5. Buck converter topology.

In Fig. 5, $S 1$ and $S 2$ represent the possible switching states of the circuit. The constant DC voltage from the supply is given by $v_{s}$, and its current by $i_{s}(t)$. The values of the inductance, capacitance and resistance are given by $L, C$ and $R$, respectively. The inductor and resistor currents are given by $i_{L}$ and $i_{R}$. Finally, the voltage at the resistance load is given by $v_{R}$.

Since the voltages and currents $v_{R}, v_{s}, i_{s}(t), i_{L}$ and $i_{R}$ are real-valued functions, we will use the $L_{2 \mathbb{R}}^{1}\left[0, T_{p w m}\right]$ space. Where $T_{p w m}$ is the time period of the Pulse Width Modulator (PWM).

To model the Buck converter depicted in Fig. 5. It is used the average model in steady state [27]. Thus, the model is given by,

$$
\begin{array}{r}
D v_{s}=v_{R}, \\
i_{L}=i_{R} .
\end{array}
$$

Where $D \in[0,1]$ is the duty cycle of the PWM, and the voltage and current values $v_{s}, v_{R}, i_{L}$ and $i_{R}$ are constant over the time interval $t \in\left[0, T_{\text {pwm }}\right]$.

With the current equation (95), it is obtained the power supply current $i_{s}(t)$, and it is depicted in Fig. 6 .

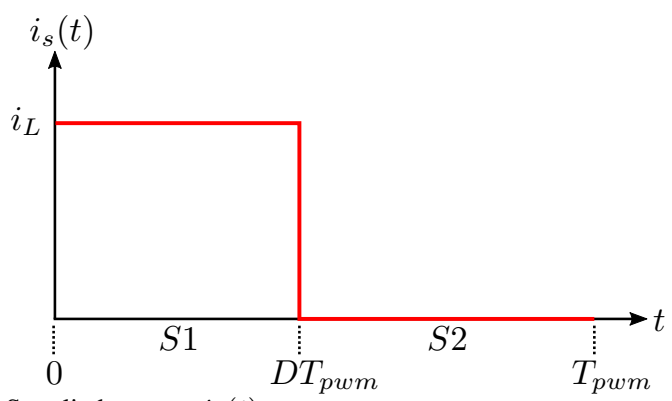

Fig. 6. Supplied current $i_{s}(t)$.

In Fig. 6, we can see that the current $i_{s}(t)$ is not proportional to the constant voltage $v_{S}$. Thus, from (49) we know that $i_{s}(t)$ is not optimal in the sense of the Least effort problem (46). To further verify this, it is used the Cauchy-Schwarz inequality (58), where the inner product $P_{S B}$ and the multiplication of the norms $S_{S B}$ of $v_{S}$ and $i_{S}(t)$ are given by,

$$
\begin{array}{r}
P_{S B}=v_{s} i_{L} D, \\
S_{S B}=v_{s} i_{L} \sqrt{D} .
\end{array}
$$

Here, the inner product $P_{S B}$ is the generalized active power, and the multiplication of the norms $S_{S B}$ is the generalized apparent power.

Thus, by writing the Cauchy-Schwarz inequality for the power supply of the Buck converter $P_{S B} \leq S_{S B}$, and eliminating $v_{s}$ and $i_{L}$. It is obtained the duty cycle inequality,

$$
D \leq \sqrt{D} .
$$

The duty cycle inequality (98) is true for the whole domain of $D$. The relation (98) is turned into an equality at the points where the Buck converter is not behaving as a switching circuit $D \in\{0\} \cup\{1\}$, and it is a strict inequality when the converter is acting as a switching circuit $D \in(0,1)$.

Therefore, when $D \in(0,1)$, the current at the power supply $i_{s}(t)$ is not optimal in the sense of the Least effort problem (46). This means that the term that measures the defect in the Cauchy-Schwarz inequality (18) in the $L_{2 \mathbb{R}}^{1}\left[0, T_{\text {pwm }}\right]$ space is 
not zero, and from (57), this term is the generalized reactive power.

\section{CONCLUSION}

In this work, it was obtained the definition of the generalized reactive power by using a Least effort problem in a real Hilbert space. It allowed us to prove that the power theory is just a geometrical property of a real Hilbert space, and that the reactive power is a scaled distance to an optimal current. Where if we do not know the direction of the current to be compensated, knowing the value of the reactive power is useless in the non-sinusoidal case. Thus, if we compensate for the current orthogonal to the voltage, we attain an optimum, liberating ampacity in the network and transmitting the same average power.

After that, we compared the power theory developed here against some other power theories. Where it was demonstrated that some of them capture the phenomena just in particular cases, and that the Budeanu's power theory is incomplete, because we do not know the direction of the current orthogonal to the voltage.

Then, with the definition of the complexification of a real Hilbert space, we revisited a computation done by B. Tellegen in a sinusoidal AC system. Where he used phasors to compute the power triangle of the load. Here, we proved that the imaginary part of the inner product in the complexification of the real Hilbert space can not be interpreted as a power. Because it belongs to the term that is measuring the defect in the Cauchy-Schwarz inequality. Thus, the reactive power shall not be interpreted as a power but rather as a measurement of the defect in the Cauchy-Schwarz inequality. Other thing that we obtained from here, is that the reactive power is not conserved in the sense of Tellegen, unless the system is working in the purely sinusoidal case.

Simultaneously, we used the power theory developed here to extend the computations to switched DC circuits. This is done because DC signals also belong to the real Hilbert spaces given in this work.

Finally, with the theory given here, we can answer the question given by C. P. Steinmetz in 1892, that began one of the biggest puzzles in Electrical Engineering: does a phase shift occur in an AC arc? The answer is no, there is no phase shift. But since the current and the voltage from the AC electric arc do not have a scalar relation given by (49), we will obtain that the active power is lower than the apparent power $P_{\mathcal{H}}<S_{\mathcal{H}}$. Thus, the angle that was thought as a phase shift by the Classical power theory is interpreted now as the angle between two vectors belonging to a real Hilbert space.

\section{ACKNOWLEDGMENT}

The author would like to thank Dr. Jonathan C. MayoMaldonado for introducing him to the problem, and Jose L. German-Felix for his useful comments.

\section{REFERENCES}

[1] C. P. Steinmetz and G. Kaiser, "Does a phase shift occur in an AC arc?(Translated version)," pp. 1-3, 2016.
[2] C. Budeanu, Puissances réactives et fictives. Impr. Cultura nationala, 1927.

[3] S. Fryze, "Moc rzeczywista, urojona i pozorna w obwodach elektrycznych o przebiegach odkształconych prąadu i napięcia," Tech. Rep. 7-8, Warsaw, 1931.

[4] H. Akagi, Y. Kanazawa, and A. Nabae, "Instantaneous Reactive Power Compensators Comprising Switching Devices without Energy Storage Components," IEEE Transactions on Industry Applications, vol. IA-20, no. 3, pp. 625-630, 1984.

[5] L. S. Czarnecki, "On Some misinterpretation of the Instantaneous Reactive Power p - q Theory," IEEE Transactions on Power Electronics, vol. 19, no. 3, pp. 828-836, 2004.

[6] G. Benysek and M. Pasko, Power Theories for Improved Power Quality. Springer, 2012

[7] W. Shepherd and P. Zakikhani, "Suggested Definition of Reactive Power for Nonsinusoidal Systems," in Proceedings of the Institution of Electrical Engineers, no. 9, pp. 1361-1362, 1972.

[8] M. Depenbrock, "The FBD-Method, a generally applicable tool for analyzing power relations," IEEE Transactions on Power Systems, vol. 8 , no. 2, pp. 381-387, 1993.

[9] L. S. Czarnecki, "Currents' Physical Components (CPC) concept: A fundamental of power theory," in ISNCC 2008: 9th Conference-Seminar, Proceedings of the International School on Nonsinusoidal Currents and Compensation, pp. 1-11, 2008.

[10] A. E. Emanuel, Power Definitions and the Physical Mechanism of Power Flow. IEEE Press, Wiley, 2010.

[11] N. Kuster and W. Moore, "On the Definition of Reactive Power Under Non-Sinusoidal Conditions," IEEE Transactions on Power Apparatus and Systems, vol. PAS-99, no. 5, pp. 1845-1854, 1980.

[12] P. Tenti and P. Mattavelli, "A Time-Domain Approach to Power Term Definitions under Non- Sinusoidal Conditions," in 6th Int. Workshop on Power Definitions and Measurement under Non-Sinusoidal Conditions, vol. 81, pp. 75-84, 2003.

[13] H. Kim and H. Akagi, "The Instantaneous Power Theory on the Rotating p-q-r Reference Frames," in IEEE 1999 International Conference on Power Electronics and Drive Systems, pp. 422-427, 1999.

[14] H. Lev-Ari and A. M. Stanković, "Instantaneous power quantities in polyphase systems - A geometric algebra approach," in 2009 IEEE Energy Conversion Congress and Exposition, ECCE 2009, pp. 592-596, 2009.

[15] A. E. Emanuel, "Summary of IEEE standard 1459: Definitions for the measurement of electric power quantities under sinusoidal, nonsinusoidal, balanced, or unbalanced conditions," IEEE Transactions on Industry Applications, vol. 40, no. 3, pp. 869-876, 2004.

[16] J. L. Willems, "The IEEE Standard 1459: What and why?," AMPS 2010 - 2010 IEEE International Conference on Applied Measurements for Power Systems, Proceedings, no. August, pp. 41-46, 2010.

[17] N. Lawhite and M. D. Ilić, "Vector space decomposition of reactive power for periodic nonsinusoidal signals," IEEE Transactions on Circuits and Systems I: Fundamental Theory and Applications, vol. 44, no. 4, pp. 338-346, 1997.

[18] H. Lev-Ari and A. Stankovic, "Hilbert space techniques for modeling and compensation of reactive power in energy processing systems," IEEE Transactions on Circuits and Systems I: Fundamental Theory and Applications, vol. 50, no. 4, pp. 540-556, 2003.

[19] A. N. Kolmogorov and S. Fomin, Elements of the Theory of Functions and Functional Analysis, Vol. II, Measure.The Lebesgue Integral. Hilbert Space. Martino Publishing, 2012.

[20] C. N. Dorny, A Vector Space Approach to Models and Optimization. John Wiley \& Sons, Inc, 1975.

[21] D. G. Luenberger, Optimization by Vector Space Methods. John Wiley \& Sons, Inc, 1969.

[22] E. García-canseco, R. Griñó, R. Ortega, and M. Salichs, "A framework for analysis and design in the nonlinear nonsinusoidal case," IEEE Control Systems Magazine, no. April, pp. 46-59, 2007.

[23] M. J. Steele, The Cauchy-Schwarz Master Class: An Introduction to the Art of Mathematical Inequalities. Cambridge, 2005.

[24] E. Kreyszig, Introductory Functional Analysis with Applications. New York: Wiley, 1989.

[25] J. C. Robinson, An Introduction to Functional Analysis. Cambridge: Cambridge University Press, 2020.

[26] B. D. H. Tellegen, "A General Network Theorem, With Applications," Philips Research Reports, vol. 7, pp. 259-269, 1952.

[27] R. W. Erickson and D. Maksimovic, Fundamentals of Power Electronics. Kluwer Academic Publisher, second ed., 2001. 